\title{
Analytical solution for reactive solute transport considering incomplete mixing within a reference elementary volume
}

\author{
Gabriele Chiogna ${ }^{1}$ and Alberto Bellin ${ }^{1}$ \\ Received 16 April 2012; revised 14 March 2013; accepted 17 March 2013; published 28 May 2013.
}

[1] The laboratory experiments of Gramling et al. (2002) showed that incomplete mixing at the pore scale exerts a significant impact on transport of reactive solutes and that assuming complete mixing leads to overestimation of product concentration in bimolecular reactions. Successively, several attempts have been made to model this experiment, either considering spatial segregation of the reactants, non-Fickian transport applying a Continuous Time Random Walk (CTRW) or an effective upscaled time-dependent kinetic reaction term. Previous analyses of these experimental results showed that, at the Darcy scale, conservative solute transport is well described by a standard advection dispersion equation, which assumes complete mixing at the pore scale. However, reactive transport is significantly affected by incomplete mixing at smaller scales, i.e., within a reference elementary volume (REV). We consider here the family of equilibrium reactions for which the concentration of the reactants and the product can be expressed as a function of the mixing ratio, the concentration of a fictitious non reactive solute. For this type of reactions we propose, in agreement with previous studies, to model the effect of incomplete mixing at scales smaller than the Darcy scale assuming that the mixing ratio is distributed within an REV according to a Beta distribution. We compute the parameters of the Beta model by imposing that the mean concentration is equal to the value that the concentration assumes at the continuum Darcy scale, while the variance decays with time as a power law. We show that our model reproduces the concentration profiles of the reaction product measured in the Gramling et al. (2002) experiments using the transport parameters obtained from conservative experiments and an instantaneous reaction kinetic. The results are obtained applying analytical solutions both for conservative and for reactive solute transport, thereby providing a method to handle the effect of incomplete mixing on multispecies reactive solute transport, which is simpler than other previously developed methods.

Citation: Chiogna, G., and A. Bellin (2013), Analytical solution for reactive solute transport considering incomplete mixing within a reference elementary volume, Water Resour. Res., 49, 2589-2600, doi:10.1002/wrcr.20200.

\section{Introduction}

[2] The fate of contaminants in heterogeneous aquifers is controlled by the complex interplay between physical and (bio)chemical processes occurring in the subsurface at a hierarchy of scales. Hydrodynamic dispersion occurring at the pore-scale influences (bio)geochemical processes through mixing, while variability at larger scales controls plume's deformation and feedbacks to mixing through the gradient of solute concentration [e.g., Kapoor and Kitanidis, 1998; Cirpka et al., 2008]. In applications, mixing is hampered due to the difficulty that bacteria or

\footnotetext{
${ }^{1}$ Department of Civil, Environmental and Mechanical Engineering, University of Trento, Trento, Italy.

Corresponding author: G. Chiogna, Department of Civil, Environmental and Mechanical Engineering, University of Trento, via Mesiano 77, Trento 38123, Italy. (gabriele.chiogna@ing.unitn.it)

reactants encounter in invading the pores and interacting with a contaminant. This represents a serious limitation to the effectiveness of natural attenuation and engineered remediation technologies.

[3] At the pore scale, flow obeys to the Navier-Stokes $(\mathrm{N}-\mathrm{S})$ equation and molecular diffusion is the key physical process which leads to mixing and therefore to attenuation of solute concentration or reaction among aqueous species, in case of multispecies reactive transport. Solutions of the $\mathrm{N}-\mathrm{S}$ equation, coupled with the transport equation, have been used to analyze incomplete mixing at the pore scale, often by using idealized pore geometries [e.g., Tartakovsky et al., 2009]. However, solving the N-S equation is not a viable solution at local or larger scales because, apart from the fact that the real geometry of the pores is unknown, the computational effort would become rapidly unbearable at scales larger than a few decimeters, e.g., at the Darcy scale. Consequently, to overcome these difficulties, upscaling procedures have been developed to model processes and interpret experimental results at larger scales [see e.g., Bear, 1972; Whitaker, 1999; Wood, 2009]. 
[4] In this context, reactive solute transport is typically modeled by the (upscaled) Advection Dispersion Reaction Equation (ADRE):

$$
\begin{aligned}
& \frac{\partial C_{j}(\mathbf{x}, t)}{\partial t}+v_{i}(\mathbf{x}, t) \frac{\partial C_{j}(\mathbf{x}, t)}{\partial x_{i}}=\frac{\partial}{\partial x_{i}}\left[D_{i k} \frac{\partial C_{j}(\mathbf{x}, t)}{\partial x_{k}}\right]+f_{j} r_{j}(\mathbf{x}, t), \\
& j=1, \ldots, n_{s}
\end{aligned}
$$

where $C_{j}$ is the concentration, $f_{j}$ is the stoichiometric coefficient and $r_{j}$ is the reaction rate of the aqueous specie $j$, and $n_{s}$ is the total number of aqueous species reacting upon mixing. In addition, $t$ is time, $\mathbf{x}$ is the spatial coordinate in the space of dimension $m$ with components $x_{i}, i=1, \ldots, m$, $v_{i}$ is the $i$-th component of the velocity field, $D_{i k}$, $k=1, \ldots, m$, are the components of the local dispersion tensor, which is assumed here to be the same for all the aqueous species. In general, the components $D_{i k}$ of the local dispersion tensor have been shown to depend on the magnitude of the local velocity $v=|\mathbf{v}|=\sqrt{\sum_{i=1}^{m} v_{i}^{2}}[$ Saffman, 1960; Pfankuch, 1963] and parametrize the effects of mixing processes occurring at the pore scale but observed at the larger continuum scale. Hereafter, Darcy and continuum scales will be considered as synonyms and will be used interchangeably.

[5] Equation (1) is obtained by imposing mass conservation of the aqueous species $j$ under the assumption that the porous media can be represented as a continuum at the Darcy scale, i.e., the scale at which hydraulic properties such as hydraulic conductivity $K$ and porosity are defined [Bear, 1972; Dagan, 1989]. This simplified approach assumes implicitly complete mixing of aqueous species within the pores at scales smaller than the Darcy scale. From a modeling and computational point of view this is a very convenient assumption.

[6] Recent theoretical [Battiato and Tartakovsky, 2011] and experimental [e.g., Gramling et al., 2002] investigations have indicated that the interplay between mixing processes and reaction time scales are generally complex and require a more sophisticated approach to be handled properly. Furthermore, Edery et al. [2012] highlighted the importance of small-scale fluctuations for reactive transport in porous media both considering particle tracking methods and the classical continuum approach, which is a standard among practitioners. Several experimental and modeling studies have investigated the role of the nonlinear interaction between hydrodynamic and (bio)geochemical processes across scales on the transport of reactive solutes. For example, Knutson et al. [2007] compared continuum and pore-scale models of biodegradation showing the importance of mixing, while Kapoor et al. [1997] and Tartakovsky et al. [2009] showed that equation (1) overestimates reaction when mixing is incomplete. The intrinsic limitations of the continuum approach to model reactive transport have been also discussed by Edery et al. [2012] and Willingham et al. [2008], among others. Deviations between upscaled parameters obtained under the hypothesis of complete mixing at the pore scale and experimental results were also found in laboratory bench-scale experiments [e.g., Klenk and Grathwohl, 2002; Levy and Berkowitz, 2003; Chiogna et al., 2010].
[7] At the laboratory scale the impact of incomplete mixing has been clearly evidenced in the experiment conducted by Gramling et al. [2002]. In this experiment, the colorimetric reaction between sodium EDTA $\left(\mathrm{Na}_{2} \mathrm{EDTA}^{2-}\right)$ and copper sulfate $\left(\mathrm{CuSO}_{4}\right)$ was imaged with a camera. These two solutes react upon mixing producing copper EDTA $\left(\mathrm{CuEDTA}^{2-}\right)$ according to the following reaction:

$$
\mathrm{CuSO}_{4}+\mathrm{Na}_{2} \mathrm{EDTA}^{2-} \rightarrow \mathrm{CuEDTA}^{2-}+2 \mathrm{Na}^{+}+\mathrm{SO}_{4}^{2-} .
$$

[8] The chamber was first saturated with a $0.02 \mathrm{M}$ solution of $\mathrm{Na}_{2} \mathrm{EDTA}^{2-}$ (clear), which successively was displaced by a $0.02 \mathrm{M}$ solution of $\mathrm{CuSO}_{4}$ (light blue). The reaction (2) occurred within the mixing layer across the boundary between the two aqueous species creating dark blue CuEDTA ${ }^{2-}$. Due to its very high reaction rate constant $\left(2.3 \times 10^{9} \mathrm{M}^{-1} \mathrm{~s}^{-1}\right)$ the reaction between the two aqueous species can be considered as instantaneous. Gramling et al. [2002] conducted also nonreactive experiments at three flow rates by flushing the glass chamber with a solution of 0.01 $\mathrm{M}$ of CuEDTA ${ }^{2-}$ (dark blue). Successively, they fitted equation (1) with $j=1$ and $r_{1}=0$ to the experimental concentration profile along the chamber considering the longitudinal dispersion coefficient $D_{l}\left[L^{2} T^{-1}\right]$ as fitting parameter. With this minimal fitting, equation (1) was able to reproduce accurately the concentration distribution of CuEDTA ${ }^{2-}$ along the chamber for the three flow rates. All the other parameters needed to solve equation (1) for the non reactive tracer are known from the experimental setup and are reported in the caption of Table 1, which shows the values of $D_{l}$ providing the best fit for the three flowrates reported in Gramling et al. [2002].

[9] The striking result of this work, however, is that the model (1) was unable to reproduce the concentration of CuEDTA $^{2-}$ in the reactive experiment, when the dispersion coefficients obtained from the nonreactive experiment were used. In particular, the concentration of the product showed a peak smaller (by about $20 \%$ ) than that predicted by the model (1) in all the snapshots showed in Figure 5 of Gramling et al. [2002], thereby suggesting, consistently with the findings of Raje and Kapoor [2000], that incomplete mixing limits the colorimetric bimolecular reaction occurring in the glass chamber.

[10] This outcome triggered an interesting discussion about the relevance of incomplete mixing at the pore scale [e.g., Jose and Cirpka, 2004] and subsequently attempts have been made to quantitatively reproduce the results of

Table 1. Hydrodynamic Parameters of the Experiments as Reported by Gramling et al. [2002]

\begin{tabular}{lccc}
\hline & \multicolumn{3}{c}{ Flow Rate $Q\left(\mathrm{ml} \mathrm{min}^{-1}\right)$} \\
\cline { 2 - 4 } Parameter & 2.67 & 16 & 150 \\
\hline$v\left(\mathrm{~cm} \mathrm{~s}^{-1}\right)$ & $1.21 \times 10^{-2}$ & $8.32 \times 10^{-2}$ & $6.70 \times 10^{-1}$ \\
$D_{l}\left(\mathrm{~cm}^{2} \mathrm{~s}^{-1}\right)$ & $1.75 \times 10^{-3}$ & $1.45 \times 10^{-2}$ & $1.75 \times 10^{-1}$ \\
$\alpha_{l}(\mathrm{~cm})$ & 0.145 & 0.174 & 0.261 \\
\hline
\end{tabular}

${ }^{\mathrm{a}}$ The column is $36 \mathrm{~cm}$ high, $5.5 \mathrm{~cm}$ large and $1.8 \mathrm{~cm}$ width and it was filled with glass bits which average size is of $0.13 \mathrm{~cm}$, resulting in a porosity $n$ of 0.36. The bulk diffusion coefficient for $\mathrm{CuEDTA}^{2-}$ is $\mathcal{D}=7.02 \times$ $10^{-7} \mathrm{~cm}^{2} \mathrm{~s}^{-1} . D_{l}$ is the hydrodynamic dispersion coefficient and $\alpha_{l}=D_{l} / v$ is the dispersivity. 
these experiments. For example, Rubio et al. [2008] suggested to solve numerically equation (1) by considering the segregation effects discussed by Kapoor et al. [1997] and using different time intervals for advection and reaction, depending on the reaction kinetic. On the other hand, Edery et al. [2009, 2010], fitted a Continuous Time Random Walk (CTRW) model to the concentration distribution of the product at a given early time and used it to successfully predict the concentration at later times. An advantage of CTRW is that it is based on a transition probability of particles which does not separate the effect of varying velocities (at all scales) into an advective and a dispersive term [Berkowitz et al., 2006] but, on the other hand, it provides only the ensemble average of the concentration. In addition, CTRW requires two adjustable parameters, as pointed out by Sanchez-Villa et al. [2010]: One parameter controls the shape of the probability density function $(p d f)$ of the particle transition times, and the other the effective reaction radius that identifies the volume of interaction surrounding the particles of each species. According to this scheme, two particles of different species react instantaneously when one enters into the interaction volume of the other; the two reacting particles are then replaced by a particle of product, which assumes the position of one of the two particles and does not react. Three adjustable parameters were used by Sanchez-Villa et al. [2010] in order to quantitatively capture the experimental results using a continuum approximation with a time-dependent reaction kinetic. Two parameters were used to define a time-dependent reaction rate, while the remaining parameter was the longitudinal dispersion coefficient. However, the latter was proved to be the least sensitive parameter. The approach adopted by Sanchez-Villa et al. [2010] was recently supported by theoretical developments of upscaling of bimolecular homogeneous irreversible reactions [Porta et al., 2012]. Another interesting description of the experiment was suggested by Oates [2007] in his PhD Thesis. He proposed to explicitly consider incomplete mixing at the pore scale by using a Beta $p d f$ for the concentration and a mixing line approach to solve the reactive problem. His model requires that two quantities are determined: The variance growth scale and the variance length scale, which were obtained by fitting the model's result to the space-time evolution of the variance of the conservative tracer and to the mean product concentration. His approach consists in solving numerically the two transport equations for the concentration mean and variance developed by Kapoor and Gelhar [1994a, 1994b] by using small perturbation approximation (in the concentration) and a Fickian closure. In the numerical solution the effect of molecular diffusion is neglected and variance destruction is controlled by the function proposed in the work by Kapoor and Kitanidis [1998]. Oates [2007] used this model to interpret an extended dataset of experiments, including his laboratory experiments and those of Raje and Kapoor [2000], but for what concerns the experiment of Gramling et al. [2002], he limited himself to a general statement that the agreement between this model and the experimental results was satisfactory.

[11] All these models have been applied with extensive fitting to experimental concentration profiles of both the nonreactive solute and the product of the reaction. Our objective here is to develop a methodology which can be applied with a minimal fitting to experimental data, thereby resulting in an improved prediction capability with respect to the aforementioned approaches. In the present work, we show that model (1) can be used to solve multispecies reactive transport problems under incomplete mixing conditions by considering solute concentration unevenly distributed within the REV according to a Beta distribution function. This approach is along the lines suggested by Oates [2007], who also assumed that point concentration is distributed in space according to the Beta distribution model, but offers the advantage of a minimal fitting to experimental results, and removes the rather limiting assumptions that the effect of molecular diffusion is negligible. In addition, we do not rely on the numerical solution of partial differential equations, but we approach the problem using analytical solutions. This is achieved by employing in the reactive transport model only parameters obtained from conservative experiments, such as the longitudinal dispersion coefficient reported in Table 1, and the measured product peak concentration at a single snapshot. Furthermore, we aim at showing that considering for the concentration variance the power law decay suggested by Fedotov et al. [2005], which is typical for decaying turbulence, suffices to reproduce accurately incomplete mixing in porous media. Therefore, differently from the approach proposed by Oates [2007], the variance decay proposed by Fedotov et al. [2005] does not require that the transport process is dominated by advection and it could be also integrated into non-Fickian transport models. We consider the reaction instantaneous, as suggested by Gramling et al. [2002], and derive from simple analytical expressions the parameters required to describe the uneven distribution of solute concentration within a REV.

\section{Theoretical Background}

[12] In this section, we briefly summarize the hypotheses we introduce to model the experiment conducted by Gramling et al. [2002]. The objective of our analysis is to implement into a continuum Darcy scale reactive transport model the effect of incomplete mixing at the pore scale. Although an extension of the present methodology to transport of reactive solutes at larger scales can be envisioned, this is beyond the scopes of the present work and will not be pursued here.

\subsection{Reactive Transport}

[13] A useful method to model reactive solute transport for an instantaneous complete bimolecular reaction can be found, for example, in Gramling et al. [2002] and Cirpka and Valocchi [2007]. This method is based on chromatographic theory [e.g., Hellferich and Klein, 1970; Valocchi et al., 1981] and can be applied when the reactants and the reaction product share the same seepage velocity and the same aqueous diffusion coefficients (conditions actually met in the experiments of Gramling et al. [2002]). Chiogna et al. [2011a] and Rolle et al. [2012] showed the relevance of molecular and pore diffusion in order to correctly model mixing for conservative as well as for reactive solute transport, both under diffusion and advection dominated flow conditions. Therefore, neglecting pore diffusion in the variance destruction term, is an important limitation of the 
model proposed by Oates [2007], which we aim to remove with our approach.

[14] Under the aforementioned hypotheses, the linear combination of the two ADREs obtained by specializing the equation (1) for one of the reactants and the product, respectively, produces an equation for a transformed total concentration which assumes the form of an ADE. By repeating this procedure with the other reactant and the product we obtain the following total concentrations of the reacting species both satisfying the standard ADE:

$$
\begin{aligned}
& A^{\mathrm{tot}}=A+\frac{f_{A}}{f_{C}} C, \\
& B^{\mathrm{tot}}=B+\frac{f_{B}}{f_{C}} C,
\end{aligned}
$$

where $f_{A}, f_{B}$, and $f_{C}$ are the stoichiometric coefficients of the aqueous species $A, B$, and $C$, respectively.

[15] In the experimental setup of Gramling et al. [2002], the compound $A\left(\mathrm{CuSO}_{4}\right)$ is introduced into the glass chamber with inlet concentration $A_{\text {in }}$ and displaces compound $B$ (EDTA ${ }^{4-}$ ), with ambient concentration $B_{\text {amb. A convenient }}$ way to treat this problem is by resorting to the concept of mixing ratio $X$ [e.g., Cirpka and Valocchi, 2007], which is defined as a normalized concentration representing the volumetric ratio of the source-related water in the mixture with the ambient solution. It is therefore possible to express $A^{\text {tot }}$ and $B^{\text {tot }}$ as a function of the mixing ratio $X$ and of the inlet and ambient concentrations as follows:

$$
\begin{gathered}
A^{\text {tot }}=X A_{\text {in }}, \\
B^{\text {tot }}=(1-X) B_{\mathrm{amb}} .
\end{gathered}
$$

where $X$ varies between 0 and 1 and represents the fraction of source water in the ambient water. Given that for an instantaneous reaction, the following condition applies:

$$
A \cdot B=0,
$$

it is possible to compute the concentrations $A$ and $B$ of the two aqueous species as a function of the mixing ratio $X$ and of the concentrations of the inlet and ambient waters, as described in Cirpka and Valocchi [2007]:

$$
\left.\begin{array}{c}
A=X \cdot A_{\mathrm{in}}-(1-X) \frac{f_{A}}{f_{B}} \cdot B_{\mathrm{amb}} \\
B=0 \\
C=(1-X) \frac{f_{C}}{f_{B}} \cdot B_{\mathrm{amb}} \\
A=0 \\
B=(1-X) \cdot B_{\mathrm{amb}}-X \cdot \frac{f_{B}}{f_{A}} \cdot A_{\text {in }} \\
C=X \cdot \frac{f_{C}}{f_{A}} \cdot A_{\text {in }}
\end{array}\right\} \text { for } X>X_{\text {crit }}
$$

where

$$
X_{\text {crit }}=\frac{f_{A} \cdot B_{\mathrm{amb}}}{f_{B} \cdot A_{\mathrm{in}}+f_{A} \cdot B_{\mathrm{amb}}},
$$

is the critical mixing ratio, i.e., the value of $X$ at which the concentrations of the species $A$ and $B$ are both zero.

[16] The mixing ratio $X$ is a conservative quantity [Cirpka and Valocchi, 2007] which in our case obeys to the classical ADE (i.e., equation (1) for $j=1$ and $r_{1}=0$ ). The approach presented in the present work, however, is general and can be applied also with other, more sophisticated, models of the space-time evolution of the mixing ratio, such as CTRW. At the Darcy scale the solution of equation (1) for a homogeneous one-dimensional (1-D) semi-infinite domain with continuous injection of the tracer at $x=0$ reads as:

$$
\bar{X}(x, t)=\frac{1}{2} \operatorname{erfc}\left(\frac{x-v \cdot t}{\sqrt{4 \cdot D_{l} \cdot t}}\right),
$$

where the overbar indicates the average over an REV, $D_{l}\left[L^{2} T^{-1}\right]$ is the longitudinal dispersion coefficient, i.e., the sum of a pore diffusion term depending on molecular diffusion and a mechanical dispersion term depending on $v\left[L T^{-1}\right]$, which is the mean seepage velocity. If the reactants are uniformly distributed within the REV equations (3)-(9) can be applied also to the mean quantities (e.g., $\bar{X}$, $\bar{A}, \bar{B}$, etc.) defined at the Darcy scale. Strictly speaking, equation (10) can be applied to interpret column experiments if the following condition is satisfied: $L>>P e d$, where $L$ is the column length, $P e$ is the Péclet number and $d$ is the average grain size [Salles et al., 1993]. Gramling et al. [2002] showed that, although this condition is not satisfied in their experiments, equation (10) provides a satisfying reproduction of the concentration profiles after tuning the dispersion coefficient. A relatively small mismatch was observed at the tails of the concentration profiles, which reduces with time (Figure 4 of Gramling et al. [2002]).

[17] In the simple case of complete mixing the substitution of equation (10) into equations (8) provides the concentrations of the three aqueous species at the continuum scale. As discussed in section 1 this leads to a significant overestimation of the concentration of the product $C$. As argued by Gramling et al. [2002] this is due to the assumption of complete mixing at the pore scale. Consequently, we hypothesize, along with Oates [2007], that a suitable model of incomplete mixing will permit to better simulate the amount of product $C$ produced by the bimolecular reaction.

\subsection{Modeling Incomplete Mixing}

[18] In order to consider incomplete mixing in modeling transport of aqueous species reacting upon mixing, we assume that: (i) at the Darcy scale the concentration of a conservative solute is well represented by the continuum model (1) with $j=1$ and $r_{1}=0$, whose solution for the 1-D case considered in the present work is given by equation (10); (ii) the reactive species share the same aqueous diffusion coefficient; iii) the reaction is instantaneous and complete; iv) within the REV, at scales not reproduced by the continuous Darcy-scale model, the mixing ratio $X$ is distributed according to the Beta $p d f$ whose mean $\bar{X}$ matches the concentration provided by equation (10). The first three assumptions are also required for the analytical approach presented in section 2.1, by assuming complete mixing [Gramling et al., 2002; Cirpka and Valocchi, 2007]. Furthermore, we neglect 
sorption because not relevant in the analyzed experiments, but in other cases chromatographic mixing may play an important role and should be taken into account [e.g., Jose and Cirpka, 2004; Oya and Valocchi, 1997].

[19] The key hypothesis, for which we seek an experimental validation with the data presented by Gramling et al. [2002], is that representing the variability of $X$ within the REV by the Beta model suffices to properly model multispecies solute reactions at the Darcy scale. This conjecture is supported by the experimental findings of Oates [2007], who showed that the pore-scale distribution (i.e., at a scale smaller than the REV) of the concentration of a passive solute in laboratory experiments is very well approximated by a Beta distribution, which requires the time evolution of the concentration mean and variance. Our work, which differs from that of Oates [2007] in the way these two concentration moments are modeled, provides a new analytical solution and a simple framework for the prediction of reactants concentrations under the aforementioned hypotheses.

[20] The Beta model has been recently applied to a wide class of transport problems of conservative as well as reactive solutes [e.g., Fedotov et al., 2005; Bellin and Tonina, 2007; Bellin et al., 2011]. It is defined as follows:

$$
p(X)=\frac{\Gamma(\phi+\psi)}{\Gamma(\phi) \Gamma(\psi)} X^{\phi-1}(1-X)^{\psi-1},
$$

where $\phi$ and $\psi$ are two positive shape parameters and $\Gamma(w)=\int_{0}^{\infty} r^{w-1} e^{-r} \mathrm{~d} r$ is the gamma function. This model has the advantage to be defined in a bounded support $(0,1)$ which spans all the possible values of $X$ and that in the absence of mixing reduces to the exact binary distribution $p(X)=\delta(1-X)(1-\bar{X})+\delta(X) \bar{X}$ as shown by Bellin and Tonina [2007].

[21] The parameters $\phi$ and $\psi$ of the Beta $p d f$ are related to the mean $\bar{X}$ and variance $\sigma_{X}^{2}$ of $X$ as follows:

$$
\begin{gathered}
\bar{X}=\int_{0}^{1} X p(X) \mathrm{d} X=\frac{\phi}{\phi+\psi}, \\
\sigma_{X}^{2}=\int_{0}^{1}(X-\bar{X})^{2} p(X) \mathrm{d} X=\frac{\phi \psi}{(\phi+\psi)^{2}(\phi+\psi+1)},
\end{gathered}
$$

and therefore:

$$
\begin{gathered}
\phi=\bar{X}\left(\frac{\bar{X}(1-\bar{X})}{\sigma_{X}^{2}}-1\right), \\
\psi=(1-\bar{X})\left(\frac{\bar{X}(1-\bar{X})}{\sigma_{X}^{2}}-1\right),
\end{gathered}
$$

where $\sigma_{X}^{2}$ is bounded between 0 and 0.25 . To simplify the notation we omitted in the expressions from (11) to (15) the dependence on space and time of $X, \bar{X}$, and consequently of $\phi$ and $\psi$.

[22] A first condition we impose to our Beta model is that the mean value of $X$ equals the corresponding value obtained by equation (10) at each position $x$ along the column. This leads to:

$$
\bar{X}(x, t)=\frac{1}{2} \operatorname{erfc}\left(\frac{x-v \cdot t}{\sqrt{4 \cdot D_{l} \cdot t}}\right)=\frac{\phi(x, t)}{\phi(x, t)+\psi(x, t)},
$$

which should be supplemented by another condition in order to obtain the parameters $\phi$ and $\psi$.

\subsection{Spatio-Temporal Evolution of the Variance of the Concentration pdf}

[23] The moments of $X$ are variable in both space and time [e.g., Fedotov et al., 2005; Kapoor and Kitanidis, 1998]. In section 2.2, we presented a model for the mean $\bar{X}$ under the assumption that it represents the concentration of a nonreactive solute evaluated at the continuum scale and here we assume, pending verification, that the variance of $X$ assumes the following form:

$$
\sigma_{X}^{2}(x, t)=g(t) \sigma_{X \text { adv }}^{2}(x, t),
$$

where $\sigma_{X \text { adv }}^{2}$ is the variance for the pure advective case, i.e., in the absence of diffusion, which assumes the following exact expression [Dagan, 1982]:

$$
\sigma_{X \mathrm{adv}}^{2}(x, t)=\bar{X}(x, t)(1-\bar{X}(x, t)) .
$$

[24] Notice that according to equation (18) $\sigma_{X \text { adv }}^{2}$ is maximum for $\bar{X}=0.5$.

[25] The dimensionless function $g$ is bounded between 0 and 1 , with the former value approached at large times when the plume is well mixed and hence $\sigma_{X}^{2} \rightarrow 0$. On the other hand, $g$ can not be larger than 1 because the concentration variance is bounded from above by $\sigma_{X \text { adv }}^{2}$. In the present work, we assume that $g$ declines proportionally to $1 / t$, according to the following expression:

$$
g(t, v)=\frac{n_{0}}{n_{0}+\frac{t v}{d}}
$$

where $n_{0}$ is the number of pore channels crossed after a time $t_{0}$ since injection (i.e., $n_{0}=t_{0} v / d$ ). For a time $t<<t_{0}$ the variance assumes the value characteristic of a purely advective process, such that $t_{0}$ can be interpreted as the time below which diffusion is negligible with respect to advection.

[26] This model of variance decay for a nonreactive solute transported in a disordered velocity field has been justified statistically through the law of large numbers (LLN) [Fedotov et al., 2005]. In fact, it is assumed that the concentration of a random conserved solute can be described as a sample mean, i.e., it converges to its mean value while its variance decays approximately as $t^{-1}$. Furthermore, this power law decay shares the same asymptotic behavior with the model proposed by Kapoor and Kitanidis [1998] for a 0 -D system, which is the dimensionality of our subgrid model. Power law decay of the variance is typical of turbulence, for example. Hence we aim at verifying if this model can also be applied to mixing processes in porous media, where the disorder comes from the complex geometry of the flow domain, rather than turbulence. Notice that, differently from the variance decay model proposed by Oates [2007], the proposed time evolution of the concentration variance does not require that transport is dominated by advection. 
[27] Substituting equations (19) and (18) into equation (17), we obtain the following expression of the concentration variance:

$$
\sigma_{X}^{2}(x, t)=\frac{n_{0}}{n_{0}+\frac{t v}{d}}(\bar{X}(x, t)(1-\bar{X}(x, t))) .
$$

[28] This model of $\sigma_{X}^{2}$ satisfies the constraints $\psi \geq 0$ and $\phi \geq 0$, as can be verified by substituting equation (20) into equations (14) and (15). An important characteristic of our model is that the concentration variance decreases monotonically with time from the maximum value observed at the initial time. A monotonically decreasing variance leads to a monotonically increasing entropy, as required by the irreversible nature of the process [Kitanidis, 1994].

\subsection{Calibration and Solution of the Reactive Transport Model}

[29] We solve the reactive transport problem under the following assumptions: (i) the spatial and temporal evolution of $\bar{X}$ and $\sigma_{X}^{2}$ are given by equations (10) and (20), respectively; (ii) the reaction is instantaneous, irreversible and complete, such that concentrations of the reaction partners and the product can be expressed through equation (8) as a function of the mixing ratio $X$.

[30] The main difference between our approach and that of Gramling et al. [2002] and Cirpka and Valocchi [2007] is that we consider $X$ variable within the REV with the constraint that its mean value is provided by equation (10), while they assumed $X$ uniform within the REV. In view of the probabilistic interpretation of the distribution of $X$ within the REV, equation (8) can be reinterpreted stochastically, with the mean concentrations of the three aqueous species assuming the following expressions:

$$
\begin{aligned}
\bar{A}(x, t)= & \int_{X_{\text {crit }}}^{1}\left(X \cdot A_{\text {in }}-(1-X) \frac{f_{A}}{f_{B}} \cdot B_{\text {amb }}\right) p(X) \mathrm{d} X, \\
\bar{B}(x, t)= & \int_{0}^{X_{\text {crit }}}\left((1-X) \cdot B_{\text {amb }}-X \cdot \frac{f_{B}}{f_{A}} \cdot A_{\text {in }}\right) p(X) \mathrm{d} X, \\
\bar{C}(x, t)= & \int_{0}^{X_{\text {crit }}}\left(X \cdot \frac{f_{C}}{f_{A}} \cdot A_{\text {in }}\right) p(X) \mathrm{d} X \\
& +\int_{X_{\text {crit }}}^{1}\left((1-X) \frac{f_{C}}{f_{B}} \cdot B_{\text {amb }}\right) p(X) \mathrm{d} X .
\end{aligned}
$$

[31] We propose to compute the parameter $n_{0}$ controlling the decay of the mixing ratio variance by imposing that the peak concentration of the product, as predicted by our model, equals the peak concentration in one of the profiles recorded during the laboratory experiment. The profile is recorded at time $t_{\text {cal }}$, while the concentration of the product reaches the peak at $x=x_{p}$ where, according to equations (16) and (20), the mean and the variance of the mixing ratio assume the following expressions:

$$
\bar{X}\left(x_{p}, t_{\mathrm{cal}}\right)=\frac{\phi\left(x_{p}, t_{\mathrm{cal}}\right)}{\phi\left(x_{p}, t_{\mathrm{cal}}\right)+\psi\left(x_{p}, t_{\mathrm{cal}}\right)},
$$

and

$$
\sigma_{X}^{2}(x, t)=\frac{n_{0}}{n_{0}+\frac{t v}{\mathrm{~d}}}\left(\bar{X}\left(x_{p}, t_{\mathrm{cal}}\right)\left(1-\bar{X}\left(x_{p}, t_{\mathrm{cal}}\right)\right)\right),
$$

respectively.
[32] Then, considering equation (21), we obtain:

$$
\begin{aligned}
\bar{C}_{\text {peak }}\left(x_{p}, t_{\text {cal }}\right) & =\int_{0}^{X_{\text {crit }}}\left(X \cdot \frac{f_{C}}{f_{A}} \cdot A_{\text {in }}\right) p(X) \mathrm{d} X \\
& +\int_{X_{\text {crit }}}^{1}\left((1-X) \frac{f_{C}}{f_{B}} \cdot B_{\text {amb }}\right) p(X) \mathrm{d} X .
\end{aligned}
$$

[33] By substituting into equation (24) the expression (11) of the pdf $p$ with the parameters $\phi$ and $\psi$ given by the equations (14) and (15), respectively, and the moments given by the equations (22) and (23), we obtain an algebraic expression in the only unknown $n_{0}$.

[34] This marks a significant difference with respect to the solution proposed by Oates [2007]. He solves numerically the two differential equations proposed by Kapoor and Gelhar [1994a]: One for the mean and the other for the variance of the mixing ratio. In particular, he obtains the parameters of the function controlling the destruction term in the differential equation for the variance by fitting the model to the concentration profiles of the product at all available times, after assuming that the mixing ratio is distributed according to the Beta distribution. Differently, we solve only an algebraic equation resulting from imposing the reproduction of the peak concentration of product extracted from the concentration profile at a given (single), known, time. This allows to obtain the parameter $n_{0}$ by using a minimal amount of information on the evolution of the product concentration along the column, while the longitudinal dispersion coefficient is obtained from independent conservative experiments. Notice that, differently from Oates [2007], who used all the available information on the nonreactive and reactive solute transport for calibrating the model, with our approach we can predict the profiles of all the aqueous species also at times different from $t_{\text {cal }}$ at which the peak concentration of the product has been detected.

[35] In the experimental setup of Gramling et al. [2002] $f_{A}=f_{B}=f_{C}$, and $A_{\text {in }}=B_{\text {amb }}$, such that $X_{\text {crit }}=0.5$. To calibrate the model we consider the experiment conducted with $Q=2.67 \mathrm{ml} \mathrm{min}^{-1}$, which at time $t=619 \mathrm{~s}$ showed the normalized peak concentration of the product $\bar{C}_{\text {peak }}=0.291$. With these parameters we obtained $n_{0}=18.5$, which corresponds to $t_{0}=199 \mathrm{~s}$. Successively, the profiles of the reagents and the product at all the times and considering different flow velocities can be computed by the expressions (21) where $\bar{X}$ and $\sigma_{X}^{2}$ are provided by the expressions (10) and (20), respectively.

\section{Comparison Between Experimental and Model Results}

[36] In this section, we validate our conceptual model against the experimental results of Gramling et al. [2002]. First we discuss the implications of incomplete mixing on the behavior the concentration along the column profile, then we apply the methodology presented in section 2 to compute the product concentration profiles and the total mass product for the set up of Gramling et al. [2002], and finally we perform a sensitivity analysis of our model to the input parameters.

\subsection{Quantification of Incomplete Mixing}

[37] The hypothesis driving the present work is that the smaller concentration of product observed in the 
experiment of Gramling et al. [2002], with respect to the predictions obtained by assuming complete mixing at the pore scale, is the consequence of a nonuniform distribution of the concentration of the aqueous species reacting upon mixing, and consequently of the distribution of the mixing ratio $X$ within the pores of the REV. This is equivalent to assuming that the entropy is always smaller than the maximum, which is achieved only when mixing is complete.

[38] This hypothesis is supported by the analysis of Cao and Kitanidis [1998], showing that at the fringes of a tracer plume mixing is incomplete, and by Tartakovsky et al. [2009] who showed that a large part of the inability of Darcy-scale models to reproduce the concentration distribution is due to incomplete mixing. Furthermore, mixing entropy and amount of the reaction product are strongly related to each other as shown by Chiogna et al. [2011a, 2011b].

[39] Possible descriptors of incomplete mixing, and hence concentration variability, inside the REV of volume $V$ with mean concentration $\bar{X}$, are the mixing ratio variance $\sigma_{X}^{2}[-]$ (equation (13)), the coefficient of variation $\mathrm{CV}[-]$ :

$$
\mathrm{CV}=\frac{\sqrt{\sigma_{X}^{2}}}{\bar{X}},
$$

and the reactor ratio $M[-]$ :

$$
M=\exp \left(-\frac{1}{V} \int_{V} \frac{X}{\bar{X}} \cdot \ln \frac{X}{\bar{X}} \mathrm{~d} V\right) .
$$

[40] For $X / \bar{X}<<1$, the reactor ratio, which is directly related to the Shannon entropy of $X$ [Kitanidis, 1994], can be approximated by the following expression [Kapoor and Kitanidis, 1996]:

$$
M \approx \exp \left(-\frac{\mathrm{CV}^{2}}{2}\right)
$$

[41] These descriptors have been also used, for example, by Cao and Kitanidis [1998] in their pore-scale study.

[42] In Figures 1a-1c, we show how these indicators of mixing vary along the column as a consequence of the variability of the shape parameters $\phi$ and $\psi$, and then ultimately on the spatial variability of $\bar{X}$ and $\sigma_{X}^{2}$. In Figures $1 \mathrm{~b}, 1 \mathrm{~d}$, and $1 \mathrm{f}$ we show how the same indicators vary as a function of $\bar{X}$.

[43] Not surprisingly, but worth noticing as a confirmation of the validity of our approach, incomplete mixing is observed only in a relatively narrow region at the fringe of the plume, particularly at intermediate values of the mean mixing ratio $\bar{X}$ (Figures 1a and 1b). Complete mixing, in fact, is attained where $\sigma_{X}^{2}=0, \mathrm{CV}=0$, and $M=1$ or $M=0$ (the reactor ratio is maximum where $A$ completely displaces $B$, i.e., for $\bar{X}=1$, and it decreases within the mixing region to finally reduce to 0 where $A$ is absent).

[44] In agreement with the observations of Cao and Kitanidis [1998], the concentration variance $\sigma_{X}^{2}$ is low for both
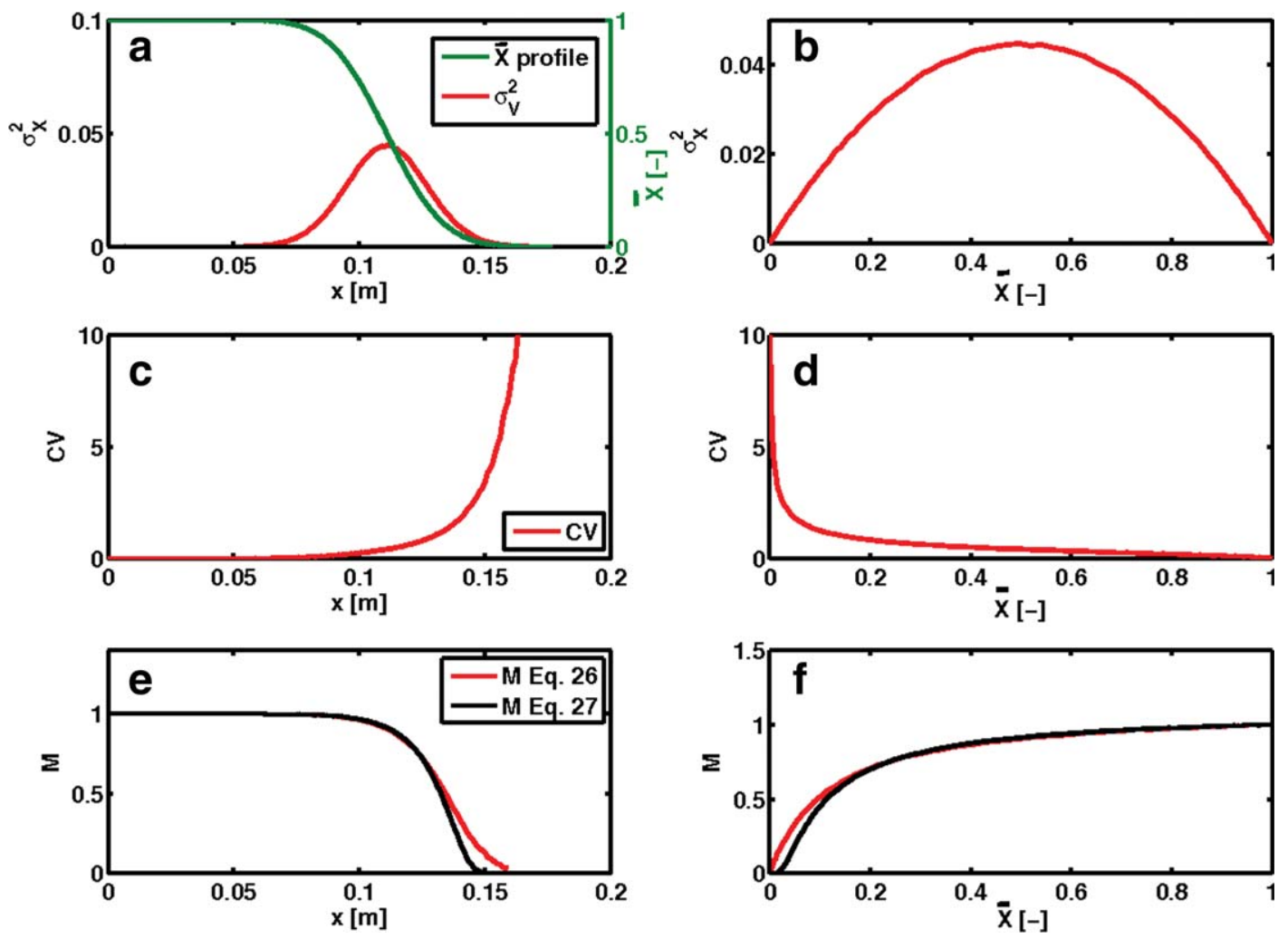

Figure 1. Concentration variance $\sigma_{X}^{2}[-]$, coefficient of variation $\mathrm{CV}[-]$ and reactor ratio $M[-]$ as a function of the distance $x[m]$ (a, c, and e, respectively) and of the mean mixing ratio $\bar{X}(\mathrm{~b}, \mathrm{~d}$, and $\mathrm{f}$, respectively) at time $t=916 \mathrm{~s}$ and flow rate $Q=2.67 \mathrm{ml} \mathrm{min}^{-1}$. In Figures $1 \mathrm{e}$ and $1 \mathrm{f}$, the approximation provided by equation (27) is also displayed with a black line. 
low and high values of $\bar{X}$. This was expected since the variability of the concentration within the REV is small close to the extreme values of the mixing ratio of 0 and 1 . The coefficient of variation (Figures $1 \mathrm{c}$ and 1d), instead, increases toward the front edge of the plume, where dilution (i.e., the reactor ratio) is lower (Figures 1e and 1f).

\subsection{Reactive Transport}

[45] Figure 2 compares the product concentration profiles obtained by applying our model to the experimental results of Gramling et al. [2002]. For the simulations we used the values of the seepage velocities and the dispersion coefficients obtained by Gramling et al. [2002] through inversion of the nonreactive tracer experiment. These parameters are reproduced in Table 1 . The modeled concentration profiles for the other times and the other flow rates are obtained without any additional fitting than the calibration procedure at time $t=916 \mathrm{~s}$. We observe that our model reproduces accurately the experimental results with only a slightly wider profile with respect to the measured data, possibly reflecting some inaccuracy of the ADE (10) to represent the mixing ratio profile. It is in fact well known that the ADE is not an exact predictor of concentration, even in the simple 1-D case considered in the present work [Berkowitz et al., 2006] and that deviations are higher, though limited, at early times. Remarkably, the accuracy of the model does not deteriorate when it is applied to reproduce concentration profiles recorded at times and flow rates not used for calibration. This reinforces our conclusion that incomplete mixing is responsible for the inability of the classic ADRE, which assumes implicitly complete mixing to reproduce the peak concentration and that our model is capable of predicting the evolution of the concentration profiles with minimal fitting performed only on one of the recorded profiles. Furthermore, although the concentration profiles for the other times and the other flow rates are obtained without fitting, they are well predicted by the model. In addition, the tails of the product concentration profiles are captured well by both the complete and the incomplete mixing models. In fact, as also pointed out by
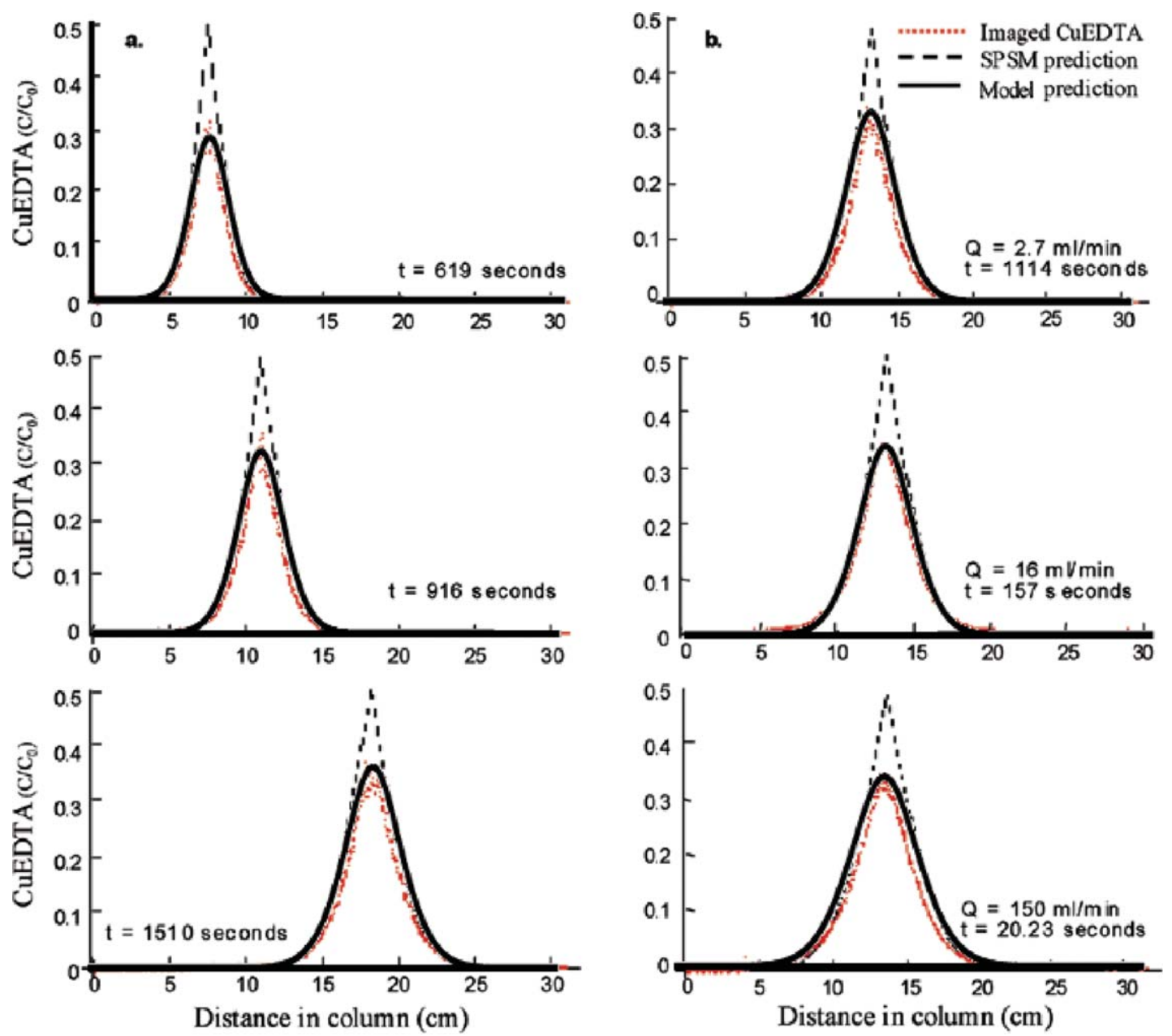

Figure 2. Product concentration profiles for (a) $Q=2.67 \mathrm{ml} \mathrm{min}^{-1}$ and three different times, including the calibration time and (b) the three flow rates used in the experiment of Gramling et al. [2002] and porosity $n=0.45$ at other times. The dotted red line represents experimental results, the black continuous line represents the incomplete mixing model while the dashed line represents the complete mixing model. 


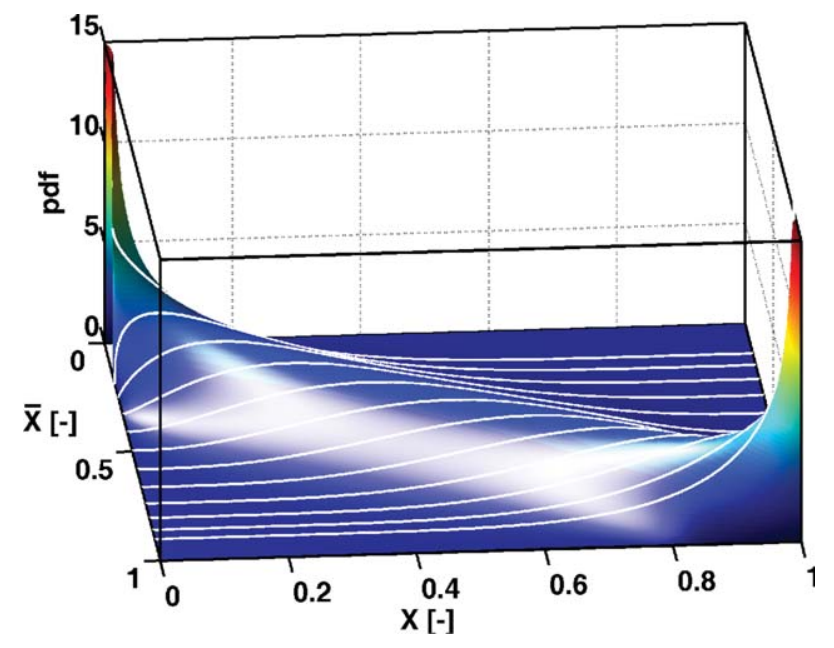

Figure 3. Probability density functions of the mixing ratio $X$ within an REV as a function of the mean value of the mixing ratio $(\bar{X}[-])$. The displayed results refer to time $t=916 \mathrm{~s}$ and flow rate $Q=2.67 \mathrm{ml} \mathrm{min}^{-1}$. The pdf has been plotted up to the value of 15 to improve readability of the graph.

Gramling et al. [2002] and shown in Figure 1, at the edges of the reaction zone the molecules of the reactants are more uniformly mixed than elsewhere.

[46] As shown in Figure 3, the $p d f$ of the concentration within the REV has two peaks at $\bar{X}=0$, and $\bar{X}=1$. This effect is determined by the physical constraint that the extreme values of $\bar{X}$ are observed where the concentration of the invading solution $A$ is uniformly equal to either 1 or 0 . Furthermore, we remark that the Beta model varies in space and time according to the mean $\bar{X}$ and the variance $\sigma_{X}^{2}$ given by equations (16) and (20), respectively.

[47] Figure 4 depicts the time evolution of mass of product produced during the experiment and predicted by our model assuming complete and incomplete mixing as a function of the cumulative output flux normalized with respect to the pore volume. The complete mixing model overpredicts the mass of product, as evidenced also by Gramling et al. [2002], Rubio et al. [2008], Edery et al. [2009, 2010], and Sanchez-Villa et al. [2010], while our incomplete mixing model produces a very good match with the experimental data at intermediate and late times. At early times, when the product is generated at short distances from the column inlet our model limits mixing more than needed, thereby resulting in a small to medium underestimation of the generated mass of product. This effect may be the consequence of minimizing mixing at early times during calibration. In making this comparison, however, it is important to consider that in the experimental procedure applied by Gramling et al. [2002] the correction used to remove the effects of light absorbance tends to overestimate the concentration product. Therefore, since the experimental error bounds are not available, it is impossible to perform a quantitative analysis on the tendency of our model to underestimate the mass of product generated at early times. Furthermore, the model and the experimental results are in agreement after more than 0.2 pore
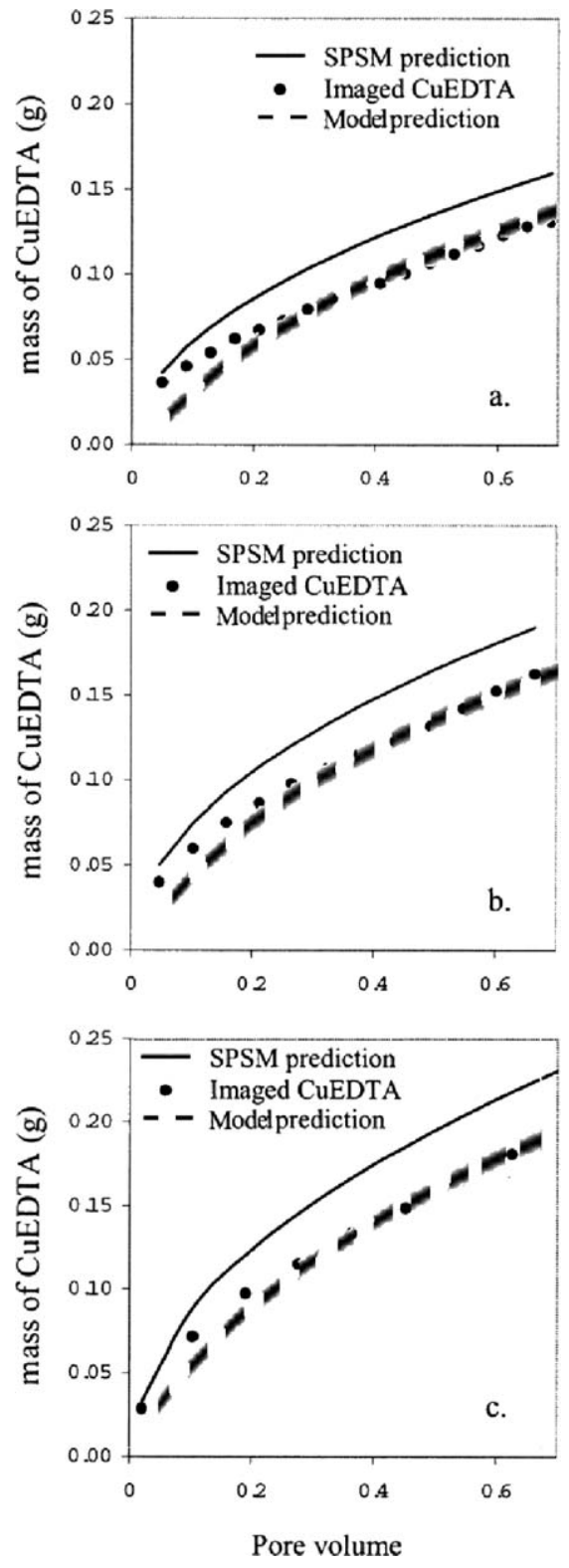

Figure 4. Total mass of product as a function of the pore volume at a flow rate of (a) $Q=2.67 \mathrm{ml} \mathrm{min}^{-1}$, (b) $Q=16 \mathrm{ml} \mathrm{min}^{-1}$, and (c) $Q=150 \mathrm{ml} \mathrm{min}^{-1}$. Experimental results are indicated by dots, while complete and incomplete mixing models are indicated by solid and dashed black lines, respectively.

volumes have flown through the column, which means that the model loses accuracy when the invading solution of $A$ has traveled less than about 60 grain diameters. This deviation may also suggest that 60 grain diameters are not enough for letting the equation (10) to represent accurately the mean concentration of a nonreactive tracer over the $\mathrm{REV}$, thereby leading to an underestimation of mixing.

\subsection{Sensitivity Analysis}

[48] In Figure 5 we show the sensitivity of the parameter $t_{0}$ to the peak concentration of the product, which is used to calibrate the model. In addition, we also show that the 

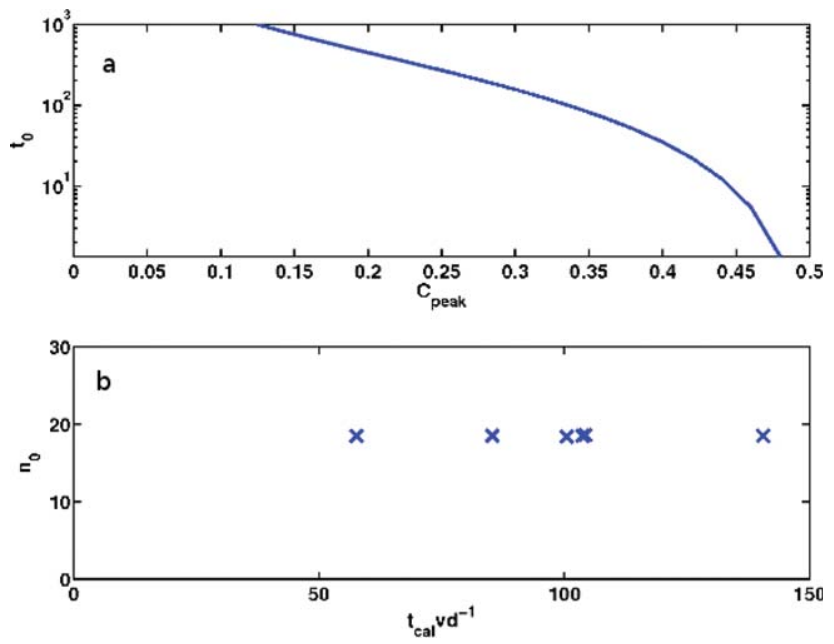

Figure 5. (a) Value of $t_{0}$ computed according to equation (24), considering a variable $\bar{C}_{\text {peak }}$ for a given time and $X_{\text {crit }}=0.5$; (b) Values of $n_{0}$ calibrating the model for the different experimental curves reported in Figure 2.

computed $n_{0}$ is the same for all the concentration profiles, including those not used for calibration. As expected for $\bar{C}_{\text {peak }} \rightarrow 0.5$ we obtain $t_{0} \rightarrow 0$, which leads to a zero concentration variance and hence to the complete mixing model result. Lower values of $\bar{C}_{\text {peak }}$ lead to high values of $t_{0}$, which means that the system is poorly mixed and that the condition of uniform concentration of the reactants within the REV will be reached only at large times.

[49] In an attempt to improve the modeling performance at early times, we calibrated again our model by considering the following generalization of the variance model (20):

$$
g(t, v)=\frac{n_{0}}{n_{1}+\frac{t v}{d}}
$$

where $n_{1}$ is an additional parameter, which assumes the meaning of a lower cutoff. The model (20) assumes implicitly that at $t=0$ the maximum value of the dimensionless variance is equal to $\sigma_{X}^{2}=0.25$, which corresponds to the situation in which the ambient water and the water entering through the inlet of the column are totally segregated at $t=0$, as it may be expected at the initial time. By introducing the parameter $n_{1}$, we relax this condition and allow the two waters to be partially mixed at the initial time, with $n_{1}$ controlling the amount of mixing through the reduction of the dimensionless concentration variance with respect to the value 0.25 obtained when mixing is not occurring. The calibration is now performed by imposing that our model matches the observed $\bar{C}_{\text {peak }}$ both at $t=619 \mathrm{~s}$ and $t=1510 \mathrm{~s}$, which produced the following parameters: $n_{0}=18.5, n_{1}=60$ (i.e., $t_{1}=699 \mathrm{~s}$ ). The results of this new calibration are shown in Figure 6 in terms of time evolution of the computed mass of product and in Figure 7 in term of concentration profiles. While at early times there is a significant difference between the product concentration profiles predicted by the two models, this difference reduces considerably at late times (i.e., for the times reported by Gramling et al. [2002]) when the two profiles are not significantly dif-

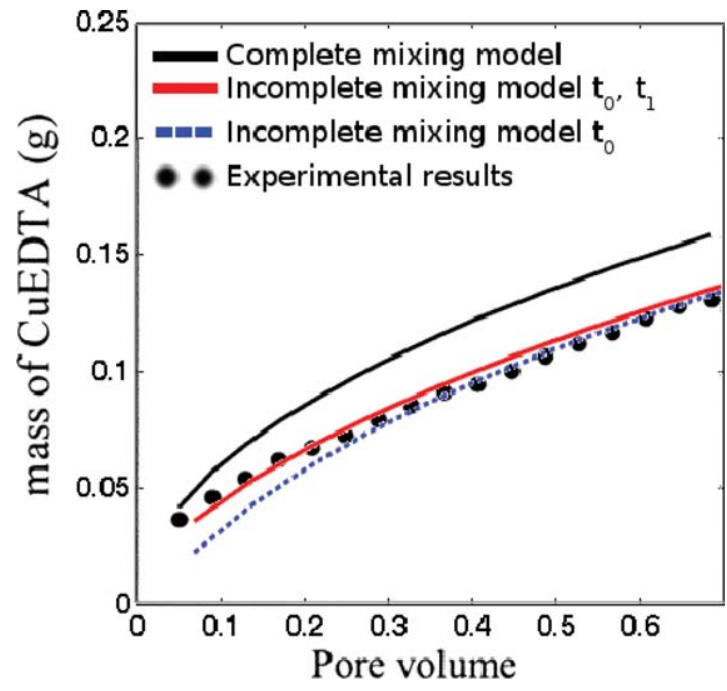

Figure 6. Total mass of product as a function of the pore volume at a flow rate $Q=2.67 \mathrm{ml} \mathrm{min}^{-1}$. The dots indicate experimental results, the solid black line the complete mixing model, the dashed blue and solid red lines are for incomplete mixing with one (i.e., $t_{0}$ ) and two (i.e., $t_{0}$ and $t_{1}$ ) fitting parameters.

ferent (see Figure 7). As shown in Figure 6 at early times the model (28) is in a better match with the recorded mass of product than the model (20), while both models are in a very good match at intermediate and late times.

[50] However, given these small differences model (28) may not be preferable to model (20) since it requires the introduction of an additional parameter, in particular if the interest of the simulations is on the intermediate and late time behavior. A more in depth analysis involving the comparison of the two models with profiles of product concentration recorded at earlier times are needed to better analyze the advantages of using the model (28) instead of the

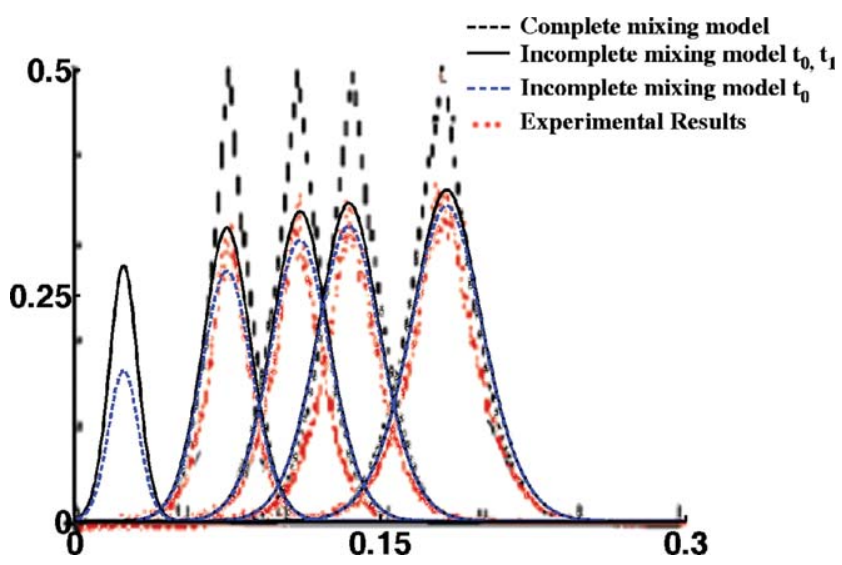

Figure 7. Product concentration profiles. The red line represents experimental results, the black continuous line represents the incomplete mixing model with one fitting parameter $\left(t_{0}\right)$, the dashed blue line represents the incomplete mixing model with two fitting parameters $\left(t_{0}\right.$ and $\left.t_{1}\right)$, while the dashed black line represents the complete mixing model. The results refer to the flow rate of $Q=2.67 \mathrm{ml} \mathrm{min}^{-1}$. 
simplest, and less demanding in terms of data for calibration, model (20). This analysis requires to compare the two models with data from several independent experiment conducted under controlled conditions and is beyond the scope of the present work.

\section{Summary and Conclusion}

[51] In the present work we have developed a new theoretical framework to model reactive transport processes in porous media considering explicitly incomplete mixing at a scale smaller than the Darcy scale. The main assumptions of this model are that (i) conservative transport is well described by a continuum Darcy-scale approach, which provides a reliable estimate of the mean mixing ratio $\bar{X}$; (ii) the reactive species have the same aqueous diffusion coefficients and seepage velocity; (iii) the reaction is instantaneous and complete; (iv) within the REV the mixing ratio $X$ is distributed according to the Beta distribution model whose mean matches the concentration at the continuum level. These hypotheses are reasonable and in line with other approaches to the same problem, [Oates, 2007; Rubio et al., 2008; Edery et al., 2009, 2010; Sanchez-Villa et al., 2010] also validated against the experiments of Gramling et al. [2002].

[52] All of them were successful in capturing the peak concentration of the product and the evolution in time of the total mass produced by the reaction. The main advantage of our model, with respect to the other models, is that it does not require a numerical solution of the reactive transport model, nor the definition of effective and time-dependent reaction parameters, since it limits the reaction describing explicitly incomplete mixing along the lines proposed by Oates [2007], but with the important differences discussed in the present work. The two shape factors of the Beta $p d f$, which characterize the amount of incomplete mixing can be derived analytically and require only the product peak concentration at a given time, while all the other parameters used in the reactive model are obtained from the concentration profile of a nonreactive tracer, which can be determined either through a tracer test or a transport model.

[53] Although the description of incomplete mixing by the use of a Beta distribution model is often encountered in the literature [e.g., Fedotov et al., 2005; Bellin and Tonina, 2007; Oates, 2007], with respect to the state of the art we coupled this model with an explicit description of the variance decay in order to model reactive transport in porous media and we provide a methodology which allows to compute reactive transport in a straightforward analytical way. In particular, the model we used to describe the variance decay is not limited to advection dominated conditions and does not require a Fickian transport assumption at short times as in previous models, such as that discussed by Oates [2007].

[54] With our analysis, we showed that the lower mass of the product, observed in the laboratory experiment of Gramling et al. [2002], with respect to that predicted by assuming complete mixing in solving equation (1), derives directly from the effect of the nonuniform distribution of the reactants within the REV. In fact, in our model, the mixing entropy of the system is lower than in case of a uniformly distributed concentration within the REV. This was shown using quantities such as the concentration variance, the coefficient of variation and the reactor ratio.

[55] The results presented in this study indicate that our approach is accurate to model homogeneous reactions in porous formations at the laboratory scale. It is straightforward to apply and less demanding in terms of calibration data than existing approaches successfully applied to the same data set we used in the present work. Although further studies are needed before applying our modeling approach under more general conditions, such as for reactants with different diffusion coefficients, or for kinetic-limited reactions and in case of heterogeneous media, it is our opinion that it provides a new, simple and effective strategy to perform reactive transport simulations with minimal fitting.

[56] Acknowledgments. This study was supported by the EU 7th Framework Programme Collaborative Research Project CLIMB (Climate Induced Changes on the Hydrology of Mediterranean Basins, grant 244151). Three anonymous reviewers and Massimo Rolle are gratefully acknowledged for providing valuable comments to the manuscript.

\section{References}

Battiato, I., and D. M. Tartakovsky (2011), Applicability regimes for macroscopic models of reactive transport in porous media, J. Contam. Hydrol., 120-121, 18-26, doi:10.1016/j.jconhyd.2010.05.005.

Bear, J. (1972), Dynamics of Fluids in Porous Media, American Elsevier, New York.

Bellin, A., and D. Tonina (2007), Probability density function of non-reactive solute concentration in heterogeneous porous formations, J. Contam. Hydrol., 94(1-2), 109-125.

Bellin, A., G. Severino, and A. Fiori (2011), On the local concentration probability density function of solutes reacting upon mixing, Water Resour. Res., 47, W01514, doi:10.1029/2010WR009696.

Berkowitz, B., A. Cortis, M. Dentz, and H. Scher (2006), Modeling nonFickian transport in geological formations as a continuous time random walk, Rev. Geophys., 44, RG2003, doi:10.1029/2005RG000178.

Cao, J., and P. K. Kitanidis (1998), Adaptive finite element simulation of Stokes flow in porous media, Adv. Water Resour., 22(1), 17-31.

Chiogna, C., O. A. Cirpka, P. Grathwohl, and M. Rolle (2011a), Relevance of local compound-specific transverse dispersion for conservative and reactive mixing in heterogeneous porous media, Water Resour. Res., 47, W07540, doi:10.1029/2010WR010270.

Chiogna, G., C. Eberhardt, P. Grathwohl, O. A. Cirpka, and M. Rolle (2010), Evidence of compound dependent hydrodynamic and (hydro)mechanical transverse dispersion by multi-tracer laboratory experiments, Environ. Sci. Technol., 44(2), 688-693, doi:10.1021/es9023964.

Chiogna, G., O. A. Cirpka, P. Grathwohl, and M. Rolle (2011b), Transverse mixing of conservative and reactive tracers in porous media: Quantification through the concepts of flux-related and critical dilution indices, Water Resour. Res., 47, W02505, doi:10.1029/2010WR009608.

Cirpka, O. A., and A. J. Valocchi (2007), Two-dimensional concentration distribution for mixing-controlled bioreactive transport in steady state, $A d v$. Water Resour., 30(6-7), 1668-1679, doi:10.1016/j.advwatres.2006.05.022.

Cirpka, O. A., R. L. Schwede, J. Luo and M. Dentz (2008), Concentration statistics for mixing-controlled reactive transport in random heterogeneous media, J. Contam. Hydrol., 98(1-2), 61-74.

Dagan, G. (1982), Stochastic modeling of groundwater flow by unconditional and conditional probabilities, 2, The solute transport, Water Resour. Res., 18(4), 835-848.

Dagan, G. (1989), Flow and Transport in Porous Formations, Springer, New York.

Edery, Y., H. Sher, and B. Berkowitz (2009), Modeling bimolecular reactions and transport in porous media, Geophys. Res. Lett., 36, L02407, doi:10.1029/2008GL036381.

Edery, Y., H. Sher, and B. Berkowitz (2010), Particle tracking model of bimolecular reactive transport in porous media, Water Resour. Res., 46, W07524, doi:10.1029/2009WR009017.

Edery, Y., A. Guadagnini, H. Sher, and B. Berkowitz (2012), Reactive transport in disordered media: Role of fluctuations in interpretation of laboratory experiments, Adv. Water. Resour., doi:10.1016/j.advwatres.2011.12.008. 


\section{CHIOGNA AND BELLIN: REACTIVE TRANSPORT AND INCOMPLETE MIXING}

Fedotov, S., M. Ihme, and H. Pitsch (2005), Stochastic mixing model with power law decay of variance, Phys. Rev. E, 71, 1-8, doi:10.1103/ PhysRevE.71.016310.

Gramling, C. M., C. F. Harvey, and L. C. Meigs (2002), Reactive transport in porous media: A comparison of model prediction with laboratory visualization, Environ. Sci. Technol., 36(11), 2508-2514.

Hellferich, F. G., and G. Klein (1970), Multicomponent Chromatography, Theory of Interference, 419 pp., Marcel Dekker, New York.

Jose, S. C., and O. A. Cirpka (2004), Measurement of mixing-controlled reactive transport in homogeneous porous media and its prediction from conservative tracer test data, Environ. Sci. Technol., 38(7), 2089-2096.

Kapoor, V., and L. W. Gelhar (1994a), Transport in three-dimensionally heterogeneous aquifers. 1. Dynamics of concentration fluctuations, Water Resour. Res., 30(6), 1775-1788.

Kapoor, V., and L. W. Gelhar (1994b), Transport in three-dimensionally heterogeneous aquifers. 2. Predictions and observations of concentration fluctuations, Water Resour. Res., 30(6), 1789-1801.

Kapoor, V., and P. K. Kitanidis (1996), Concentration fluctuations and dilution in two-dimensionally periodic heterogeneous porous media, Transp. Porous Media, 22, 91-119.

Kapoor, V., and P. K. Kitanidis (1998), Concentration fluctuations and dilution in aquifers, Water Resour. Res., 34(5), 1181-1193.

Kapoor, V., L. W. Gelhar, and F. Miralles-Wilhem (1997), Bimolecular second-order reactions in spatially varying flows: segregation induced scale-dependent transformation rates, Water Resour. Res., 33(4), 527536.

Kitanidis, P. K. (1994), The concept of the dilution index, Water Resour. Res., 30(7), 2011-2026.

Klenk, I., and P. Grathwohl (2002), Transverse vertical dispersion in groundwater and the capillary fringe, J. Contam. Hydrol., 58(1-2), 111128, doi:10.1016/S0169-7722(02)00011-6.

Knutson, C., A. Valocchi, and C. Werth (2007), Comparison of continuum and pore-scale models of nutrient biodegradation under transverse mixing conditions, Adv. Water Resour., 30(6-7), 1421-1431, doi:10.1016/ j.advwatres.2006.05.012.

Levy, M., and B. Berkowitz (2003), Measurement and analysis of nonFickian dispersion in heterogeneous porous media, J. Contam. Hydrol., 64(3-4), 203-226, doi:10.1016/S0169-7722(02)00204-8.

Oates, P. (2007), Upscaling reactive transport in porous media: laboratory visualization and stochastic models, Ph.D. thesis, Mass. Inst. Technol., USA.
Oya, S., and A. J. Valocchi (1997), Characterization of traveling waves and analytical estimation of pollutant removal in one-dimensional subsurface bioremediation modeling, Water Resour. Res., 33(5), 1117-1127.

Pfankuch, H. O. (1963), Contribution à l' étude des dèplacement de fluides miscible dans un milieu poreux, Rev. Inst. Fr. Pet., 18(2), 215-270.

Porta, G., M. Riva, and A. Guadagnini (2012), Upscaling solute transport in porous media in the presence of an irreversible bimolecular reaction, Adv. Water Resour., 35, 151-162, doi:10.1016/j.advwatres.2011.09.004.

Raje, D. S., and V. Kapoor (2000), Experimental study of bimolecular reaction kinetics in porous media, Environ. Sci. Technol., 34(7), 1234-1239.

Rolle, M., D. Hochstetler, G. Chiogna, P. Kitanidis, and P. Grathwohl (2012), Experimental investigation and pore-scale modeling interpretation of compound-specific transverse dispersion in porous media, Transp. Porous Media, 93(3), 347-362, doi:10.1007/s11242-012-9953-8.

Rubio, A., A. Zalts, and C. E. Hasi (2008), Numerical solution of the advection-reaction-diffusion equation at different scales, Environ. Model. Software, 23, 90-95.

Saffman, P. G. (1960), Dispersion due to molecular diffusion and macroscopic mixing in flow through a network of capillaries, J. Fluid Mech., $7(2), 194-208$.

Salles, J., J. Thovert, R. Delannay, L. Prevors, J. Auriault, and P. Adler (1993), Taylor dispersion in porous media. determination of the dispersion tensor, Phys. Fluids A, 5(10), 2348-2376.

Sanchez-Villa, X., D. Fernadez-Garcia, and A. Gaudagnini (2010), Interpretation of column experiments of transport of solutes undergoing an irreversible bimolecular reaction using a continuum approximation, Water Resour. Res., 46, W12510, doi:10.1029/2010WR009539.

Tartakovsky, A. M., G. D. Tartakovsky, and T. D. Scheibe (2009), Effects of incomplete mixing on multicomponent reactive transport, Adv. Water Resour., 32(11), 1674-1679, doi:10.1016/j.advwatres.2009.08.012.

Valocchi, A. J., R. L. Street, and P. V. Roberts (1981), Transport of ionexchanging solutes in groundwater: Chromatographic theory and field simulation, Water Resour. Res., 17(5), 1517-1527.

Whitaker, D. (1999), The Method of Volume Averaging, Kluwer Acad., Netherlands.

Willingham, T. W., C. J. Werth, and A. J. Valocchi (2008), Evaluation of the effects of porous media structure on mixing-controlled reactions using pore-scale modeling and micromodel experiments, Environ. Sci. Technol., 42, 3185-3193.

Wood, B. (2009), The role of scaling laws in upscaling, Adv. Water Resour., 32, 723-736, doi:10.1016/j.advwatres.2008.08.015. 